\title{
LIPSCHITZ EXTENSIONS AND APPROXIMATIONS
}

\author{
VALENTIN GUTEV
}

\begin{abstract}
The classical Hahn-Banach theorem is based on a successive pointby-point procedure of extending bounded linear functionals. In the setting of a general metric domain, the conditions are less restrictive and the extension is only required to be Lipschitz with the same Lipschitz constant. In this case, the successive procedure can be replaced by a much simpler one which was done by McShane and Whitney in the 1930s. Using virtually the same construction, Czipszer and Gehér showed a similar extension property for pointwise Lipschitz functions. In the present paper, we relate this construction to another classical result obtained previously by Hausdorff and dealing with pointwise Lipschitz approximations of semi-continuous functions. Moreover, we furnish complementary extension-approximation results for locally Lipschitz functions which fit naturally in this framework.
\end{abstract}

\section{Introduction}

Let $(X, d)$ and $(Y, \rho)$ be metric spaces. A map $f: X \rightarrow Y$ is called Lipschitz if there exists $K \geq 0$ such that

$$
\rho(f(p), f(q)) \leq K d(p, q), \quad \text { for all } p, q \in X .
$$

In this case, to emphasise on the constant $K$, we also say that $f$ is $K$-Lipschitz. The least $K \geq 0$ for which $f$ is $K$-Lipschitz is called the Lipschitz constant of $f$, and denoted by $\operatorname{Lip}(f)$. The Lipschitz constant of a map $f: X \rightarrow Y$ at a non-isolated point $p \in X$ is defined by

$$
\operatorname{Lip}(f, p)=\varlimsup_{x \rightarrow p} \frac{\rho(f(x), f(p))}{d(x, p)}=\inf _{t>0}\left[\sup _{0<d(x, p)<t} \frac{\rho(f(x), f(p))}{d(x, p)}\right] .
$$

For an isolated point $p \in X$, we simply set $\operatorname{Lip}(f, p)=0$. A map $f: X \rightarrow Y$ is called pointwise Lipschitz if $\operatorname{Lip}(f, p)<+\infty$, for every $p \in X$. For $\delta>0$, let $\mathbf{O}(p, \delta)$ be the open $\delta$-ball centred at $p$, i.e.

$$
\mathbf{O}(p, \delta)=\{x \in X: d(x, p)<\delta\} .
$$

Date: February 19, 2020.

2010 Mathematics Subject Classification. 26A16, 41A30, 54C20, 54C30, 54D20, 54E35, $54 \mathrm{E} 40$.

Key words and phrases. Lipschitz function, pointwise Lipschitz function, locally Lipschitz function, Lipschitz in the small function, extension, pointwise and uniform approximation. 
It follows from (1.2) that $f: X \rightarrow Y$ is pointwise Lipschitz if and only if for each $p \in X$ there exists $L_{p} \geq 0$ and $\delta_{p}>0$ such that

$$
\rho(f(x), f(p)) \leq L_{p} d(x, p), \quad \text { whenever } x \in \mathbf{O}\left(p, \delta_{p}\right) .
$$

Finally, let us recall that a map $f: X \rightarrow Y$ is locally Lipschitz if each point $p \in X$ is contained in an open set $U \subset X$ such that $f \uparrow U$ is Lipschitz.

There are simple examples of locally Lipschitz functions which are neither Lipschitz nor uniformly continuous. On the other hand, each locally Lipschitz map on a compact metric space is Lipschitz. In fact, it was shown by Scanlon [37, Theorem 2.1] that $f: X \rightarrow Y$ is locally Lipschitz iff its restriction on each compact subset of $X$ is Lipschitz. According to (1.4), each pointwise Lipschitz map is continuous and each locally Lipschitz map is pointwise Lipschitz. The converse is not true, see e.g. [16, Example 2.7]. Locally Lipschitz and pointwise Lipschitz functions are naturally related to differentiability. In 1919, Rademacher [35] showed that each locally Lipschitz function $f: \Omega \rightarrow \mathbb{R}$, defined on an open set $\Omega \subset \mathbb{R}^{n}$, is differentiable almost everywhere (in the sense of the Lebesgue measure). In 1923, Stepanov (spelled also Stepanoff) [38, 39] extended Rademacher's result to pointwise Lipschitz functions. In the literature, Stepanov's theorem is traditionally proved by applying Rademacher's theorem to Lipschitz extensions of Lipschitz functions; a straightforward simple proof based on Lipschitz approximations was given by J. Malý [31].

Regarding Lipschitz extensions, the following theorem was obtained by McShane [32, Theorem 1], see also Whitney [43, the footnote on p. 63], and is commonly called the McShane-Whitney extension theorem.

Theorem 1.1. Let $(X, d)$ be a metric space, $A \subset X$ and $\varphi: A \rightarrow \mathbb{R}$ be a $K$ Lipschitz function. Then there exists a K-Lipschitz function $\Phi: X \rightarrow \mathbb{R}$ which is an extension of $\varphi$, i.e. with $\Phi \uparrow A=\varphi$.

The extension in Theorem 1.1 was defined by an explicit formula involving the function $\varphi$, the Lipschitz constant $K$ and the metric $d$, see (2.3). It is interesting to compare this theorem with two other classical results. Namely, the construction in Theorem 1.1 is nearly the same as the one used for the proof of the classical Hahn-Banach theorem [5, 23], see also [6, 7, 8] and a previous work of Helly [26]. This relationship was explicitly stated by several authors, for instance in Czipszer and Gehér [11], where they rediscovered Theorem 1.1. This construction is also virtually the same as the one used by Hausdorff [24] to deal with pointwise limits of semi-continuous functions, see (2.1) and (2.2). In fact, in his proof of a more general result, see [24, page 293], Hausdorff credited the construction to Moritz Pasch and showed that it always gives rise to a Lipschitz function. In contrast to the Hahn-Banach theorem, the relationship with the Pasch-Hausdorff construction remained somehow unnoticed. 
The construction in Theorem 1.1 was further applied to pointwise Lipschitz functions by Czipszer and Gehér [11, Theorem II]. Namely, they proved the following extension result.

Theorem 1.2. If $(X, d)$ is a metric space and $A \subset X$ is a closed subset, then each pointwise Lipschitz function $\varphi: A \rightarrow \mathbb{R}$ can be extended to a pointwise Lipschitz function $\Phi: X \rightarrow \mathbb{R}$.

In some sources, Theorem 1.2 was wrongly attributed as an extension result for locally Lipschitz functions, see e.g. [10, Theorem 4.1.7]. Furthermore, in other sources, Theorem 1.2 was even used as an extension result for locally Lipschitz functions. For instance, it was applied in [37, Theorem 3.1] to show that a subset $A \subset X$ of a metric space $(X, d)$ is closed if and only if each (bounded) locally Lipschitz function $\varphi: A \rightarrow \mathbb{R}$ can be extended to a locally Lipschitz function $\Phi: X \rightarrow \mathbb{R}$. However, locally Lipschitz extensions were not discussed by Czipszer and Gehér in [11]. In fact, the author is not aware of any explicit formula that will result in locally Lipschitz extensions. Such extensions were implicitly obtained by Luukkainen and Väisälä in [29, Theorem 5.12] using a different technique. Namely, Luukkainen and Väisälä showed that if $A$ is a closed subset of a metric space $(X, d)$, then every locally Lipschitz map $\varphi: A \rightarrow M$, where $M$ is an $n$-dimensional locally Lipschitz manifold, has a locally Lipschitz extension to a neighbourhood of $A$ in $X$. Their proof was based on paracompactness of metrizable spaces, hence relying heavily on the axiom of choice.

We are now ready to state also the main purpose of this paper. Namely, in this paper we aim to fill in this gap and present simple and self-contained proofs of several extension and approximation results for Lipschitz-like functions. In the next section, we briefly discuss the Pasch-Hausdorff construction and its refinement in the setting of Theorem 1.1. In Section 3, we also illustrate how this construction can be applied to simplify the proof of Theorem 1.2. In Section 4, we show that Theorem 1.2 is still valid if "pointwise Lipschitz" is replaced by "locally Lipschitz", see Theorem 4.1. Our proof of this result is implicitly based on countable paracompactness of metrizable spaces. Briefly, using a construction of M. Mather and its refinement for countable covers in [18], we give a direct simple proof that any countable open cover of a metric space admits an index-subordinated locally finite partition of unity consisting of locally Lipschitz functions, see Proposition 4.2. This is a simplified version of a more general result obtained by Z. Frolík [19]. The last section of this paper contains several applications of the approach developed in the previous sections. For instance, we refine Haudorff's result that any bounded semi-continuous function on a metric space is a pointwise limit of some monotone sequence of Lipschitz functions. Namely, as commented in Remark 2.6, this is not true for unbounded functions. Regarding the role of boundedness, we show that each semi-continuous function defined on a 
metric space is a pointwise limit of some monotone sequence of locally Lipschitz functions (Theorem 5.1). As for semi-continuity, we generalise the well-known property that each continuous function on a metric space is a uniform limit of locally Lipschitz functions (Theorem 5.2). Finally, we also give a simple proof that each uniformly continuous function on a metric space is a uniform limit of Lipschitz in the small functions.

\section{A Construction of Lipschitz Functions}

For a metric space $(X, d)$, a function $\varphi: X \rightarrow \mathbb{R}$ is lower (upper) semicontinuous if for every $p \in X$,

$$
\varliminf_{x \rightarrow p} \varphi(x) \geq \varphi(p) \quad\left(\text { respectively, } \varlimsup_{x \rightarrow p} \varphi(x) \leq \varphi(p)\right) .
$$

Here, $\underline{\lim }_{x \rightarrow p} \varphi(x)=\sup _{\delta>0} \inf \varphi(\mathbf{O}(p, \delta))$, see (1.3).

Semi-continuity was introduced by Baire in his 1899 thesis [1], where he showed that each semi-continuous function $\varphi: \mathbb{R}^{m} \rightarrow \mathbb{R}$ is a pointwise limit of continuous functions, see also [2, 4]. Subsequently, he generalised this result in [3] by showing that a function $\varphi: \mathbb{R}^{m} \rightarrow \mathbb{R}$ is upper semi-continuous if and only if it is the pointwise limit of a decreasing sequence of continuous functions. In 1915, Tietze extended Baire's result to arbitrary metric spaces. Namely, in [41, Theorem 2], he showed that for a metric space $(X, d)$, each upper semi-continuous bounded function $\varphi: X \rightarrow \mathbb{R}$ is the pointwise limit of some decreasing sequence of continuous functions $f_{n}: X \rightarrow \mathbb{R}, n \in \mathbb{N}$. Assuming that $\varphi: X \rightarrow[\lambda, \mu]$ for some $\mu \geq \lambda>0$, Tietze defined the required functions by the following explicit formula

$$
f_{n}(p)=\sup _{x \in X} \frac{\varphi(x)}{[1+d(x, p)]^{n}}, \quad p \in X .
$$

In 1919, Hausdorff [24] gave a simple proof of this result using another direct construction, which he credited to Moritz Pasch. In fact, Hausdorff obtained the following stronger result as it is evident from his proof.

Theorem 2.1 ([24]). Let $(X, d)$ be a metric space and $\varphi: X \rightarrow \mathbb{R}$ be a function which is bounded from below. For each $\kappa>0$, define a function $f_{\kappa}: X \rightarrow \mathbb{R}$ by

$$
f_{\kappa}(p)=\inf _{x \in X}[\varphi(x)+\kappa d(x, p)], \quad p \in X .
$$

Then $f_{\kappa}$ is $\kappa$-Lipschitz. If moreover $\varphi$ is lower semi-continuous, then $\left\{f_{n}: n \in \mathbb{N}\right\}$ is an increasing sequence which is pointwise convergent to $\varphi$.

Proof. We present a brief sketch of the original proof. For $p, q, x \in X$ and $\kappa>0$, by the triangular inequality, $\kappa d(x, p) \leq \kappa d(x, q)+\kappa d(q, p)$. Hence, by adding $\varphi(x)$ to both sides and taking infimum, we get that $f_{\kappa}(p) \leq f_{\kappa}(q)+\kappa d(q, p)$. Since $d(p, q)=d(q, p)$, this is equivalent to $\left|f_{\kappa}(p)-f_{\kappa}(q)\right| \leq \kappa d(p, q)$. It is 
also evident that $f_{\kappa} \leq f_{\kappa+\varepsilon} \leq \varphi$, whenever $\varepsilon \geq 0$. Finally, for each $n \in \mathbb{N}$, take $x_{n} \in X$ with $\varphi\left(x_{n}\right)+n d\left(x_{n}, p\right)<f_{n}(p)+\frac{1}{n}$. Then $p=\lim _{n \rightarrow \infty} x_{n}$ because $d\left(x_{n}, p\right)<\frac{1}{n}\left[f_{n}(p)-\varphi\left(x_{n}\right)+\frac{1}{n}\right] \leq \frac{1}{n}\left[\varphi(p)-\inf _{x \in X} \varphi(x)+\frac{1}{n}\right]$. If $\varphi$ is lower semicontinuous, this implies that

$$
\varphi(p) \leq \varliminf_{x \rightarrow p} \varphi(x) \leq \varliminf_{n \rightarrow \infty} \varphi\left(x_{n}\right) \leq \lim _{n \rightarrow \infty} f_{n}(p) \leq \varphi(p) .
$$

Let us remark that in the original proof, see [24, page 293], Hausdorff showed that $f_{\kappa}$ is $(\kappa+1)$-Lipschitz, but the proof above is virtually the same. Here is also a simple observation relating the case of upper semi-continuous functions to that of Theorem 2.1.

Proposition 2.2. Let $(X, d)$ be a metric space and $\psi: X \rightarrow \mathbb{R}$ be a function which is bounded from above. For each $\kappa>0$, define a function $f_{\kappa}^{*}: X \rightarrow \mathbb{R}$ by

$$
f_{\kappa}^{*}(p)=\sup _{x \in X}[\psi(x)-\kappa d(x, p)], \quad p \in X .
$$

Then $f_{\kappa}^{*}=-f_{k}$, where $f_{k}$ is defined as in (2.1) with respect to the additive inverse function $\varphi=-\psi$.

The Pasch-Hausdorff construction in (2.1) and its alternative interpretation in (2.2) can be applied to partial bounded functions preserving essentially the same proof. Furthermore, they can be also applied to partial Lipschitz functions to extend them by preserving the same Lipschitz constant.

Theorem 2.3. Let $(X, d)$ be a metric space, $A \subset X$ and $\varphi: A \rightarrow \mathbb{R}$ be a $\lambda$ Lipschitz function for some $\lambda \geq 0$. Define functions $\Phi_{-}: X \rightarrow(-\infty,+\infty]$ and $\Phi_{+}: X \rightarrow[-\infty,+\infty)$ by

$$
\begin{cases}\Phi_{-}(p)=\sup _{a \in A}[\varphi(a)-\lambda d(a, p)] & \text { and } \\ \Phi_{+}(p)=\inf _{a \in A}[\varphi(a)+\lambda d(a, p)], & p \in X .\end{cases}
$$

Then $\Phi_{-} \leq \Phi_{+}$and $\Phi_{-}, \Phi_{+}: X \rightarrow \mathbb{R}$ are $\lambda$-Lipschitz extensions of $\varphi$.

Proof. Let $a, b \in A$ and $p \in X$. Since $\varphi$ is $\lambda$-Lipschitz

$$
\varphi(a)-\varphi(b) \leq \lambda d(a, b) \leq \lambda d(a, p)+\lambda d(p, b) .
$$

Accordingly, $\Phi_{-}(p) \leq \Phi_{+}(p)$ because $\varphi(a)-\lambda d(a, p) \leq \varphi(b)+\lambda d(b, p)$. By taking $p \in A$, this implies that $\varphi(p) \leq \Phi_{-}(p) \leq \Phi_{+}(p) \leq \varphi(p)$, so $\Phi_{-} \uparrow A=\varphi=\Phi_{+} \uparrow A$. In fact, the function $\Phi_{+}$corresponds to the function $f_{\lambda}$ in (2.1), the only difference is that now the infimum is taken on the points of $A$. Hence, the same argument as in Theorem 2.1 shows that it is $\lambda$-Lipschitz. Accordingly, $\Phi_{-}$is also $\lambda$-Lipschitz, see Proposition 2.2.

Remark 2.4. The Lipschitz extension $\Phi_{-}$in Theorem 2.3 represents McShane and Whitney's approach to show Theorem 1.1. The other extension $\Phi_{+}$was 
used by Czipszer and Gehér for their alternative proof of this theorem. The advantage of using both functions $\Phi_{-}$and $\Phi_{+}$, as done in Theorem 2.3, lies in the simplification of the proof. Furthermore, as pointed out implicitly in [11], $\Phi_{-} \leq f \leq \Phi_{+}$for every $\lambda$-Lipschitz extension $f: X \rightarrow \mathbb{R}$ of $\varphi: A \rightarrow \mathbb{R}$. Indeed, such a function $f$ is a $\lambda$-Lipschitz extension of itself and by Theorem 2.3,

$$
\Phi_{-}(p)=\sup _{a \in A}[\varphi(a)-\lambda d(a, p)] \leq \sup _{x \in X}[f(x)-\lambda d(x, p)]=f(p), \quad p \in X .
$$

Similarly, $f \leq \Phi_{+}$. Thus, $\Phi_{-}$is the smallest possible $\lambda$-Lipschitz extension of $\varphi$, while $\Phi_{+}$- the largest one.

Remark 2.5. For a metric space $(X, d)$, a bounded function $\varphi: X \rightarrow \mathbb{R}$ and $\kappa>0$, let $f_{\kappa}: X \rightarrow \mathbb{R}$ be defined as in (2.1). Then $f_{\kappa}$ is the greatest $\kappa$-Lipschitz function with $f_{\kappa} \leq \varphi$. Indeed, for a $\kappa$-Lipschitz function $f: X \rightarrow \mathbb{R}$ with $f \leq \varphi$, by Theorem 2.3 (applied with $A=X$ and $\varphi=f$ ),

$$
f(p)=\inf _{x \in X}[f(x)+\kappa d(x, p)] \leq \inf _{x \in X}[\varphi(x)+\kappa d(x, p)]=f_{\kappa}(p), \quad p \in X .
$$

Based on this property, the functions $f_{\kappa}, \kappa>0$, are often called the PaschHausdorff envelope of $\varphi$. One can easily see that $f=\lim _{n \rightarrow \infty} f_{n}$ is the largest lower semi-continuous function $f: X \rightarrow \mathbb{R}$ with $f \leq \varphi$. In fact, $f$ is the well-known lower Baire function associated to $\varphi$ because $f(p)=\underline{\lim }_{x \rightarrow p} \varphi(x), p \in X$.

Remark 2.6. The restriction in Theorem 2.1 that $\varphi$ is bounded from below is necessary. Indeed, let $X=\mathbb{R}$ be the real line endowed with an equivalent bounded metric, for instance $d(x, y)=\frac{|x-y|}{1+|x-y|}, x, y \in \mathbb{R}$, and $\varphi: X \rightarrow \mathbb{R}$ be the identity. Then for any $\kappa$-Lipschitz function $f: X \rightarrow \mathbb{R}$, as in Remark 2.5, it follows that

$$
\inf _{x \in X}[x+\kappa d(x, p)]=-\infty<f(p)=\inf _{x \in X}[f(x)+\kappa d(x, p)], \quad p \in X .
$$

Accordingly, the inequality $f \leq \varphi$ is impossible.

\section{Pointwise-Lipschitz Extensions}

Let $(X, d)$ and $(Y, \rho)$ be metric spaces. Following the Lipschitz condition (1.1), we shall say that a map $f: X \rightarrow Y$ is globally Lipschitz at a point $p \in X$ if there exists $K_{p} \geq 0$ such that

$$
\rho(f(x), f(p)) \leq K_{p} d(x, p), \quad \text { for every } x \in X .
$$

In this case, $K_{p}$ will be called a global Lipschitz constant at $p \in X$. Here is an example of maps which are globally Lipschitz at each point of their domain.

Proposition 3.1. If $(X, d)$ and $(Y, \rho)$ are metric spaces, then each bounded pointwise Lipschitz map $f: X \rightarrow Y$ is globally Lipschitz at each point of $X$. 
Proof. Let $M \geq 0$ be such that $\rho(f(x), f(z)) \leq M$, for all $x, z \in X$. Also, for a point $p \in X$, let $L_{p} \geq 0$ and $\delta_{p}>0$ be as in (1.4). Then $K_{p}=\max \left\{L_{p}, \frac{M}{\delta_{p}}\right\}$ is as in $(3.1)$.

Proposition 3.1 allows to apply the construction in Theorem 2.3, but now using some fixed global Lipschitz constants of a bounded pointwise Lipschitz function. Namely, let $(X, d)$ be a metric space and $A \subset X$. Whenever $\varphi: A \rightarrow \mathbb{R}$ is a bounded pointwise Lipschitz function and $\lambda_{a}, a \in A$, are fixed global Lipschitz constants of $\varphi$, following (2.3), we may associate the pair of extended functions $\Phi_{-}: X \rightarrow(-\infty,+\infty]$ and $\Phi_{+}: X \rightarrow[-\infty,+\infty)$ defined by

$$
\begin{cases}\Phi_{-}(p)=\sup _{a \in A}\left[\varphi(a)-\lambda_{a} d(a, p)\right] & \text { and } \\ \Phi_{+}(p)=\inf _{a \in A}\left[\varphi(a)+\lambda_{a} d(a, p)\right], & \text { for every } p \in X .\end{cases}
$$

In this general setting, just as in Theorem 2.3, we have that

$$
\Phi_{-} \leq \Phi_{+} \quad \text { and } \quad \Phi_{-} \uparrow A=\varphi=\Phi_{+} \uparrow A .
$$

Indeed, for $a, b \in A$, let $\lambda_{a b}=\min \left\{\lambda_{a}, \lambda_{b}\right\}$. Then for every $p \in X$, it follows from (3.1) that $\varphi(a)-\varphi(b) \leq \lambda_{a b} d(a, b) \leq \lambda_{a} d(a, p)+\lambda_{b} d(b, p)$. In other words, $\varphi(a)-\lambda_{a} d(a, p) \leq \varphi(b)+\lambda_{b} d(b, p)$ which is clearly equivalent to $\Phi_{-}(p) \leq \Phi_{+}(p)$. Similarly, taking $p \in A$, it follows from $(3.2)$ that $\varphi(p) \leq \Phi_{-}(p) \leq \Phi_{+}(p) \leq \varphi(p)$, therefore $\Phi_{-} \uparrow A=\varphi=\Phi_{+} \uparrow A$.

The approach of using both functions $\Phi_{-}$and $\Phi_{+}$is also successful in simplifying the original proof of Theorem 1.2, see [11, Theorem II]. The simplification is essentially in the following special case of this theorem.

Theorem 3.2. If $(X, d)$ is a metric space, $A \subset X$ is a closed set and $M>0$, then each pointwise Lipschitz function $\varphi: A \rightarrow(-M, M)$ can be extended to a pointwise Lipschitz function $\Phi: X \rightarrow(-M, M)$.

Proof. By Proposition 3.1, a pointwise Lipschitz function $\varphi: A \rightarrow(-M, M)$ is globally Lipschitz at each point of $A$. So, we may associate the extended functions $\Phi_{-}: X \rightarrow(-\infty,+\infty]$ and $\Phi_{+}: X \rightarrow[-\infty,+\infty)$ defined as in (3.2) with respect to some global Lipschitz constants $\lambda_{a}, a \in A$, of $\varphi$. Then by (3.3), $\Phi_{-}$and $\Phi_{+}$ are extensions of $\varphi$ with $\Phi_{-} \leq \Phi_{+}$and, in particular, usual functions. We will show that they are pointwise Lipschitz as well. Since $-\varphi: A \rightarrow(-M, M)$ is also pointwise Lipschitz, just as in Proposition 2.2, this is reduced to showing that one of these functions, say $\Phi_{+}$, is pointwise Lipschitz. So, take a point $p \in X$.

If $p \in A$ and $x \in X$, then by (3.3), $\Phi_{-}(x) \leq \Phi_{+}(x)$ and $\Phi_{+}(p)=\varphi(p)$. Hence, it follows from (3.2) that

$$
\Phi_{+}(p)-\lambda_{p} d(p, x) \leq \Phi_{-}(x) \leq \Phi_{+}(x) \leq \Phi_{+}(p)+\lambda_{p} d(p, x) .
$$

Thus, $\left|\Phi_{+}(x)-\Phi_{+}(p)\right| \leq \lambda_{p} d(p, x)$. 
Otherwise, if $p \notin A$, we will show that $\Phi_{+}$is Lipschitz in the open $\delta$-ball $\mathbf{O}(p, \delta)$, see (1.3), where $\delta=\frac{d(p, A)}{2}>0$ is the half distance between $p$ and the closed set $A$. To this end, fix a point $c \in A$ with $d(c, p) \leq 3 \delta$. Then by (3.2),

$$
\Phi_{+}(x) \leq \varphi(c)+\lambda_{c} d(c, x) \leq M+4 \delta \lambda_{c}=M_{c}, \quad \text { for all } x \in \mathbf{O}(p, \delta) .
$$

Take now $x, y \in \mathbf{O}(p, \delta)$ and any $\varepsilon>0$. By (3.2), $\Phi_{+}(x)+\varepsilon \geq \varphi(a)+\lambda_{a} d(a, x)$ for some $a \in A$. Therefore, by (3.4), $\lambda_{a} \leq \frac{\Phi_{+}(x)-\varphi(a)+\varepsilon}{d(a, x)} \leq \frac{M_{c}+M+\varepsilon}{\delta}$ because $d(a, x) \geq \delta$. For this $a \in A$, we also have that $\Phi_{+}(y) \leq \varphi(a)+\lambda_{a} d(a, y)$. Accordingly,

$$
\begin{aligned}
\Phi_{+}(y)-\Phi_{+}(x) & \leq \lambda_{a} d(y, a)-\lambda_{a} d(x, a)+\varepsilon \\
& \leq \lambda_{a} d(y, x)+\varepsilon \leq \frac{M_{c}+M+\varepsilon}{\delta} d(y, x)+\varepsilon .
\end{aligned}
$$

Thus, $\Phi_{+}(y)-\Phi_{+}(x) \leq \frac{M_{c}+M}{\delta} d(y, x)$ and, by symmetry, $\Phi_{+}$is $\operatorname{Lipschitz}$ in $\mathbf{O}(p, \delta)$.

We may now define $\Phi=\frac{\Phi_{-}+\Phi_{+}}{2+d(\cdot, A)}$, where $d(\cdot, A)$ is the distance function. Then $\Phi$ remains a pointwise Lipschitz extension of $\varphi$ because $\frac{1}{2+d(\cdot, A)}$ is a bounded Lipschitz function whose restriction on $A$ is the constant $\frac{1}{2}$. Moreover, $\Phi$ takes values in $(-M, M)$. Indeed, this is evident for the points of $A$ because $\Phi\lceil A=\varphi$. For the rest of the points of $X$, it suffices to see that $\left|\Phi_{+}(p)+\Phi_{-}(p)\right| \leq 2 M$ because $d(p, A)>0$, whenever $p \notin A$. The latter property follows easily from (3.2) because for $a \in A$, we have that $\Phi_{+}(p)+\varphi(a)-\lambda_{a} d(a, p) \leq 2 \varphi(a) \leq 2 M$. Hence, taking supremum on $A$, we get that $\Phi_{+}(p)+\Phi_{-}(p) \leq 2 M$. Similarly, we also have that $\Phi_{-}(p)+\Phi_{+}(p) \geq-2 M$.

We conclude this section with several remarks regarding the proper place of Theorem 3.2.

Remark 3.3. Theorem 1.2 follows easily from Theorem 3.2. For instance, as in [11], to a pointwise Lipschitz function $\varphi: A \rightarrow \mathbb{R}$ one can associate the function

$$
\tilde{\varphi}=\arctan \circ \varphi: A \stackrel{\varphi}{\rightarrow} \mathbb{R} \stackrel{\arctan }{\longrightarrow}\left(-\frac{\pi}{2}, \frac{\pi}{2}\right) .
$$

Then $\tilde{\varphi}$ is also pointwise Lipschitz because $\arctan : \mathbb{R} \rightarrow\left(-\frac{\pi}{2}, \frac{\pi}{2}\right)$ is itself Lipschitz. Hence, by Theorem 3.2, $\tilde{\varphi}$ has a pointwise Lipschitz extension $\tilde{\Phi}: X \rightarrow\left(-\frac{\pi}{2}, \frac{\pi}{2}\right)$. Finally, since $\tan :\left(-\frac{\pi}{2}, \frac{\pi}{2}\right) \rightarrow \mathbb{R}$ is locally Lipschitz, we may define the required extension of $\varphi$ by $\Phi=\tan \circ \tilde{\Phi}: X \rightarrow \mathbb{R}$.

Remark 3.4. One can easily see that in Theorems 1.2 and 3.2, the requirement $A$ to be closed is essential. For instance, the function $\frac{t}{|t|}:[-1,0) \cup(0,1] \rightarrow\{-1,1\}$ is even locally Lipschitz, but cannot be extended continuously on $[-1,1]$.

Remark 3.5. According to the proof of Theorem 3.2, the extensions $\Phi_{-}$and $\Phi_{+}$, defined as in (3.2), are locally Lipschitz at the points of $X \backslash A$. Our proof of this fact is essentially the same as the original proof in [11, Theorem II]. In 
contrast, the original proof that $\Phi_{+}$is pointwise Lipschitz at the points of $A$ is too complicated. The same proof of this fact was also given in [10, Theorem 4.1.7]. In both sources, for points $p \in A$ and $x \in X$, it was used the definition of $\Phi_{+}$in (3.2) to get at once that $\Phi_{+}(x)-\Phi_{+}(p) \leq \lambda_{p} d(x, p)$. However, the other inequality that $\Phi_{+}(x)-\Phi_{+}(p) \geq-\lambda_{p} d(x, p)$ was obtained by taking $a \in A \backslash\{p\}$ and manipulating the inequalities $\varphi(a)-\varphi(p) \geq-\lambda_{a} d(a, p)$ and $\varphi(a)-\varphi(p) \geq-\lambda_{p} d(a, p)$; in fact, by multiplying the first one by $\frac{d(a, x)}{d(a, x)+d(x, p)}$ and the second one by $\frac{d(x, p)}{d(a, x)+d(x, p)}$. The other benefit of using both functions $\Phi_{-}$and $\Phi_{+}$is the immediate construction of the extension $\Phi: X \rightarrow(-M, M)$. Namely, in both [11] and [10], this extension is obtained by refining the construction of $\Phi_{+}$to get a pointwise Lipschitz extension $\Psi_{+}: X \rightarrow(-M, M]$ of $\varphi$. Next, applying the same construction to $-\varphi$, to get a similar pointwise Lipschitz extension $\Psi_{-}: X \rightarrow[-M, M)$ of $-\varphi$. Then the required extension was defined by $\Psi=\frac{\Psi_{+}-\Psi_{-}}{2}$.

\section{Locally Lipschitz Extensions}

In this section, we will show the following extension theorem.

Theorem 4.1. Let $(X, d)$ be a metric space and $A \subset X$ be a closed set. Then each locally Lipschitz function $\varphi: A \rightarrow \mathbb{R}$ can be extended to a locally Lipschitz function $\Phi: X \rightarrow \mathbb{R}$.

As mentioned in the Introduction, a special case of Theorem 4.1 follows from a more general result, see [29, Theorem 5.12], which is based on paracompactness of metrizable spaces. Here, we use a similar approach but based only on countable paracompactness. Recall that a space $X$ is countably paracompact if each countable open cover of $X$ has a locally finite open refinement. The fact that each metrizable space is countably paracompact is a simple consequence of Dowker's result [14, Theorem 2], see also [36, Theorem 1.1]. Here, we will use this implicitly based on the following considerations.

The cozero set, or the set-theoretic support, of a function $\xi: X \rightarrow \mathbb{R}$ is the set $\operatorname{coz}(\xi)=\{x \in X: \xi(x) \neq 0\}$. A collection $\xi_{n}: X \rightarrow[0,1], n \in \mathbb{N}$, of continuous functions on a space $X$ is a partition of unity if $\sum_{n=1}^{\infty} \xi_{n}(x)=1$, for each $x \in X$. A partition of unity $\left\{\xi_{n}: n \in \mathbb{N}\right\}$ is locally finite if $\left\{\operatorname{coz}\left(\xi_{n}\right): n \in \mathbb{N}\right\}$ is a locally finite cover of $X$; and it is index-subordinated to a cover $\left\{O_{n}: n \in \mathbb{N}\right\}$ of $X$ if $\operatorname{coz}\left(\xi_{n}\right) \subset O_{n}$, for every $n \in \mathbb{N}$. It was shown in $[19$, Lemma] that each countable open cover of a metric space admits a locally finite index-subordinated partition of unity consisting of Lipschitz functions. Here, we give a direct proof of the following special case of this result.

Proposition 4.2. Each open cover $\left\{O_{n}: n \in \mathbb{N}\right\}$ of a metric space $(X, d)$ admits a locally finite index-subordinated partition of unity consisting of locally Lipschitz functions. 
Proof. If $X=O_{n}$ for some $n \in \mathbb{N}$, the property is trivial. Suppose that $O_{n} \neq X$ for every $n \in \mathbb{N}$. Following the proof of [19, Lemma], for each $n \in \mathbb{N}$, consider the 1-Lipschitz function $\eta_{n}: X \rightarrow\left[0,2^{-n}\right]$ defined by $\eta_{n}(x)=\min \left\{d\left(x, X \backslash O_{n}\right), 2^{-n}\right\}$, $x \in X$. Then $\operatorname{coz}\left(\eta_{n}\right)=O_{n}, n \in \mathbb{N}$, and the function $\eta=\sum_{n=1}^{\infty} \frac{\eta_{n}}{2^{n}}: X \rightarrow[0,1]$ remains 1-Lipschitz. Moreover, $\eta$ is positive-valued because $\left\{\operatorname{coz}\left(\eta_{n}\right): n \in \mathbb{N}\right\}$ is a cover of $X$. We may now use a construction M. Mather, see [12, Lemma] and [17, Lemma 5.1.8], in fact its further refinement for countable covers in [18]. Namely, for each $n \in \mathbb{N}$, define a function $\gamma_{n}(x)=\max \left\{\eta_{n}(x)-\frac{1}{2} \eta(x), 0\right\}, x \in X$. Evidently, $0 \leq \gamma_{n} \leq \eta_{n}$ and $\gamma_{n}$ is Lipschitz because so are $\eta_{n}$ and $\eta$. If $p \in X$, then $\eta(p)>2^{-k}$ for some $k \in \mathbb{N}$, hence $p$ is contained in an open set $V \subset X$ with $\eta(x)>2^{-k}$ for every $x \in X$. Accordingly, $\gamma_{n}(x)=0$ for every $x \in V$ and $n>k$, so $\left\{\operatorname{coz}\left(\gamma_{n}\right): n \in \mathbb{N}\right\}$ is locally finite. It is also a cover of $X$. Indeed, for $p \in X$, we have that $\sup _{n \in \mathbb{N}} \eta_{n}(p) \geq 2^{-n_{0}}>\eta_{n}(p)$ for some $n_{0} \in \mathbb{N}$ and every $n>n_{0}$. Therefore, $\sup _{n \in \mathbb{N}} \eta_{n}(p)=\eta_{m}(p)$ for some $m \leq n_{0}$. This implies that $\eta(p) \leq \sum_{n=1}^{\infty} \frac{\eta_{m}(p)}{2^{n}}=\eta_{m}(p)$. Accordingly, $\eta_{m}(p)>\frac{1}{2} \eta(p)$ and $\gamma_{m}(p)>0$. We may now define the required partition of unity by $\xi_{k}=\frac{\gamma_{k}}{\sum_{n=1}^{\infty} \gamma_{n}}, k \in \mathbb{N}$. It consists of locally Lipschitz functions because $\sum_{n=1}^{\infty} \gamma_{n}$ is a bounded function and locally it is a finite sum of Lipschitz functions.

Proposition 4.2 is in good accord with the following property of bounded locally Lipschitz functions.

Proposition 4.3. If $(X, d)$ and $(Y, \rho)$ are metric spaces and $f: X \rightarrow Y$ is a bounded locally Lipschitz map, then $X$ has an open increasing cover $\left\{U_{n}: n \in \mathbb{N}\right\}$ such that $f \uparrow U_{n}$ is Lipschitz, for each $n \in \mathbb{N}$.

Proof. Let $M \geq 0$ be such that $\rho(f(x), f(z)) \leq M$, for every $x, z \in X$. Also, for $p \in X$, let $\delta_{p}>0$ and $L_{p} \geq 0$ be such that $\rho(f(x), f(z)) \leq L_{p} d(x, z)$, for every $x, z \in \mathbf{O}\left(p, \delta_{p}\right)$. As in Proposition 3.1, setting $K_{p}=\max \left\{L_{p}, \frac{M}{\delta_{p}}\right\}$, it follows that

$$
\rho(f(x), f(z)) \leq K_{p} d(x, z), \quad \text { for every } x \in X \text { and } z \in \mathbf{O}\left(p, \delta_{p}\right) .
$$

Finally, set $U_{n}=\bigcup\left\{\mathbf{O}\left(p, \delta_{p}\right): K_{p} \leq n\right\}, n \in \mathbb{N}$. Evidently, $\left\{U_{n}: n \in \mathbb{N}\right\}$ is an increasing open cover of $X$. Moreover, if $x, z \in U_{n}$, then $x \in \mathbf{O}\left(p, \delta_{p}\right)$ and $z \in \mathbf{O}\left(q, \delta_{q}\right)$ for some $p, q \in X$ with $\max \left\{K_{p}, K_{q}\right\} \leq n$. According to (4.1), this implies that $\rho(f(x), f(z)) \leq n d(x, z)$.

Based on this, we have the following extension result.

Proposition 4.4. Let $(X, d)$ be a metric space and $A \subset X$ be a closed subset. Then each bounded locally Lipschitz function $\varphi: A \rightarrow \mathbb{R}$ can be extended to a locally Lipschitz function $\Phi: X \rightarrow \mathbb{R}$.

Proof. By Proposition 4.3, $A$ has an open cover $\left\{U_{n}: n \in \mathbb{N}\right\}$ such that each restriction $\varphi_{n}=\varphi\left\lceil U_{n}, n \in \mathbb{N}\right.$, is Lipschitz. Since $A$ is closed, this cover can 
be extended to an open cover $\left\{O_{n}: n \in \mathbb{N}\right\}$ of $X$, i.e. with the property that $O_{n} \cap A=U_{n}, n \in \mathbb{N}$. Then by Proposition 4.2, $\left\{O_{n}: n \in \mathbb{N}\right\}$ has an indexsubordinated locally finite partition of unity $\left\{\xi_{n}: n \in \mathbb{N}\right\}$ consisting of locally Lipschitz functions. Moreover, by Theorem 1.1, each $\varphi_{n}: U_{n} \rightarrow \mathbb{R}$ can be extended to a Lipschitz function $\Phi_{n}: X \rightarrow \mathbb{R}$. We can now define a function $\Phi: X \rightarrow \mathbb{R}$ by $\Phi(x)=\sum_{n=1}^{\infty} \xi_{n}(x) \cdot \Phi_{n}(x), x \in X$. Then $\Phi$ remains locally Lipschitz because each $p \in X$ has a neighbourhood $V \subset X$ such that $\left\{n \in \mathbb{N}: \operatorname{coz}\left(\xi_{n}\right) \cap V \neq \varnothing\right\}$ is finite, therefore $\Phi \uparrow V$ is a finite sum of locally Lipschitz functions. Moreover, $\Phi$ is an extension of $\varphi$. Indeed, take a point $a \in A$ and set $\sigma_{a}=\left\{n \in \mathbb{N}: \xi_{n}(a)>0\right\}$. Then $\Phi_{n}(a)=\varphi_{n}(a)=\varphi(a)$ for every $n \in \sigma_{a}$, and therefore

$$
\Phi(a)=\sum_{n \in \sigma_{a}} \xi_{n}(a) \cdot \Phi_{n}(a)=\varphi(a) \cdot \sum_{n \in \sigma_{a}} \xi_{n}(a)=\varphi(a) \cdot 1=\varphi(a) .
$$

Finally, we also have the following refinement of Proposition 4.4.

Corollary 4.5. If $(X, d)$ is a metric space, $A \subset X$ is a closed set and $M>0$, then each locally Lipschitz function $\varphi: A \rightarrow(-M, M)$ can be extended to a locally Lipschitz function $\Phi: X \rightarrow(-M, M)$.

Proof. If $\varphi: A \rightarrow(-M, M)$ is locally Lipschitz, then by Proposition 4.4, it can be extended to a locally Lipschitz function $\Psi: X \rightarrow \mathbb{R}$. Let $U=\Psi^{-1}((-M, M))$, which is an open set containing $A$. Hence, there is a locally Lipschitz function $\eta: X \rightarrow[0,1]$ with $\eta^{-1}(0)=X \backslash U$ and $\eta^{-1}(1)=A$. For instance, $\eta \equiv 1$ is the constant 1 if $U=X$; otherwise we can take $\eta=\frac{d(\cdot, X \backslash U)}{d(\cdot, A)+d(\cdot, X \backslash U)}$ because the distance functions $d(\cdot, X \backslash U)$ and $d(\cdot, A)$ are Lipschitz. Then the product function $\Phi=\eta \cdot \Psi: X \rightarrow(-M, M)$ is as required.

Proof of Theorem 4.1. We can proceed as in Remark 3.3. Briefly, for a locally Lipschitz function $\varphi: A \rightarrow \mathbb{R}$, the function $\tilde{\varphi}=\arctan \circ \varphi: A \rightarrow\left(-\frac{\pi}{2}, \frac{\pi}{2}\right)$ is also locally Lipschitz. Hence, by Corollary 4.5 , it can be extended to a locally Lipschitz function $\tilde{\Phi}: X \rightarrow\left(-\frac{\pi}{2}, \frac{\pi}{2}\right)$. Finally, since $\tan :\left(-\frac{\pi}{2}, \frac{\pi}{2}\right)$ is locally Lipschitz, the function $\Phi=\tan \circ \tilde{\Phi}: X \rightarrow \mathbb{R}$ is as required.

We conclude this section with the following two remarks.

Remark 4.6. The construction in Proposition 4.4 is standard and can be applied to give a direct proof of Theorem 4.1. To this end, let us recall that an arbitrary collection $\xi_{\alpha}: X \rightarrow[0,1], \alpha \in \mathscr{A}$, of continuous functions on a space $X$ is a partition of unity if $\sum_{\alpha \in \mathscr{A}} \xi_{\alpha}(x)=1$, for each $x \in X$. Here, " $\sum_{\alpha \in \mathscr{A}} \xi_{\alpha}(x)=1$ " means that only countably many functions $\xi_{\alpha}$ 's do not vanish at $x$, and the series composed by them is convergent to 1 . Now, suppose that $(X, d), A \subset X$ and $\varphi: A \rightarrow \mathbb{R}$ are as in Theorem 4.1. Then by definition, each point $a \in A$ has a neighbourhood $U_{a} \subset A$ such that the restriction $\varphi_{a}=\varphi \uparrow U_{a}$ is Lipschitz. Since $A$ is closed, one can take an open cover $\left\{O_{a}: a \in A\right\}$ of $X$ such that $O_{a} \cap A=U_{a}$, 
$a \in A$. Also, using Theorem 1.1, one can extend each $\varphi_{a}: U_{a} \rightarrow \mathbb{R}$ to a Lipschitz function $\Phi_{a}: X \rightarrow \mathbb{R}$. Thus, the proof is reduced to the existence of a locally finite partition of unity $\left\{\xi_{a}: a \in A\right\}$ which is index-subordinated to $\left\{O_{a}: a \in A\right\}$ and consists of locally Lipschitz functions. The construction of such a partition of unity is not a simple fact and will require the use of A. H. Stone's theorem [40, Corollary 1] that each metrizable space is paracompact, also the Lefschetz lemma [28] that each point-finite open cover of a normal space has a closed shrinking. However, both these results rely heavily on the axiom of choice. That is, this approach will make the proof unnecessarily complicated.

Remark 4.7. It was shown by Dowker [14, Theorem 4] that a space $X$ is countably paracompact and normal if and only if for each pair of functions $\varphi, \psi: X \rightarrow \mathbb{R}$ such that $\varphi$ is upper semi-continuous, $\psi$ is lower semi-continuous and $\varphi<\psi$, there exists a continuous function $f: X \rightarrow \mathbb{R}$ with $\varphi<f<\psi$. For a metric space $(X, d)$, this result was partially refined in [29, Theorem 5.4] by showing that $f$ can be constructed to be locally Lipschitz. The argument suggested in [29] is to follow an alternative proof of this theorem given in [15, 4.3, p. 171] which is based on paracompactness and partitions of unity, but now to take a locally Lipschitz partition of unity. In this regard, let us explicitly remark that the locally Lipschitz partitions of unity considered in [29] are based on paracompactness of metrizable spaces, see Remark 4.6. Using Proposition 4.2, one can get a simple direct proof of this fact. Briefly, as in [15], take an open cover $\left\{O_{r}: r \in \mathbb{Q}\right\}$ of $X$ such that $\varphi(x)<r<\psi(x)$, for every $x \in O_{r}$ and $r \in \mathbb{Q}$. Since the rational numbers $\mathbb{Q}$ are countable, by Proposition 4.2, $\left\{O_{r}: r \in \mathbb{Q}\right\}$ has an index-subordinated locally finite partition of unity $\left\{\xi_{r}: r \in \mathbb{Q}\right\}$ consisting of locally Lipschitz functions. The required locally Lipschitz function $f: X \rightarrow \mathbb{R}$ is now defined by $f(x)=\sum_{r \in \mathbb{Q}} \xi_{r}(x) \cdot r, x \in X$, see the proof of Proposition 4.4.

\section{Pointwise and Uniform Approximations}

As shown in Remark 2.6, the approximation property in Theorem 2.1 is not valid for unbounded functions. For such functions, using the method developed in the previous section, we have the following refined property.

Theorem 5.1. If $(X, d)$ is a metric space, then each lower semi-continuous function $\varphi: X \rightarrow \mathbb{R}$ is a pointwise limit of some increasing sequence of locally Lipschitz functions.

Proof. Let $\varphi: X \rightarrow \mathbb{R}$ be lower semi-continuous. Then for each $k \in \mathbb{N}$, the set $O_{k}=\{x \in X: \varphi(x)>-k\}$ is open. Moreover, $X=\bigcup_{k=1}^{\infty} O_{k}$ and by Proposition 4.2, there exists a locally finite partition of unity $\left\{\xi_{k}: k \in \mathbb{N}\right\}$ of locally Lipschitz functions such that $\operatorname{coz}\left(\xi_{k}\right) \subset O_{k}, k \in \mathbb{N}$. On the other hand, we may define a sequence $\varphi_{k}: X \rightarrow \mathbb{R}, k \in \mathbb{N}$, of (bounded from below) lower semi-continuous 
functions by

$$
\varphi_{k}(x)= \begin{cases}\varphi(x) & \text { if } x \in O_{k} \\ -k & \text { otherwise }\end{cases}
$$

Then by Theorem 2.1, each $\varphi_{k}$ is the pointwise limit of some increasing sequence $f_{n}^{k}: X \rightarrow \mathbb{R}, n \in \mathbb{N}$, of Lipschitz functions. We may now define the required sequence of functions $f_{n}: X \rightarrow \mathbb{R}, n \in \mathbb{N}$, by $f_{n}(x)=\sum_{k=1}^{\infty} \xi_{k}(x) \cdot f_{n}^{k}(x), x \in X$. Indeed, as in the proof of Proposition 4.4, each $f_{n}$ is locally Lipschitz. It is also evident that $f_{n} \leq f_{n+1}$ because $f_{n}^{k} \leq f_{n+1}^{k}$, for every $k \in \mathbb{N}$. Finally, take a point $p \in X$ and set $\sigma_{p}=\left\{k \in \mathbb{N}: \xi_{k}(p)>0\right\}$. Then $f_{n}(p)=\sum_{k \in \sigma_{p}} \xi_{k}(p) \cdot f_{n}^{k}(p)$ and by (5.1), $\lim _{n \rightarrow \infty} f_{n}^{k}(p)=\varphi(p)$ for every $k \in \sigma_{p}$. Since $\sigma_{p}$ is a finite set, this implies that $\lim _{n \rightarrow \infty} f_{n}(p)=\sum_{k \in \sigma_{p}} \xi_{k}(p) \cdot \varphi(p)=\varphi(p) \cdot 1=\varphi(p)$.

In case the function $\varphi: X \rightarrow \mathbb{R}$ in Theorem 5.1 is continuous (i.e. both lower and upper semi-continuous), it can be uniformly approximated by locally Lipschitz functions. The following result is an alternative version of [29, Theorem 5.17] which doesn't make use of paracompactness of metrizable spaces.

Theorem 5.2. Let $(X, d)$ be a metric space, $\varphi: X \rightarrow \mathbb{R}$ and $\varepsilon: X \rightarrow(0,+\infty)$ be continuous functions, and $A \subset X$ be a closed set. Then each locally Lipschitz function $g: A \rightarrow \mathbb{R}$ with $|g(a)-\varphi(a)|<\varepsilon(a), a \in A$, can be extended to a locally Lipschitz function $f: X \rightarrow \mathbb{R}$ such that $|f(x)-\varphi(x)|<\varepsilon(x)$, for every $x \in X$.

Regarding the proper place of Theorem 5.2, let us remark that the range in [29, Theorem 5.17] is an $n$-dimensional locally Lipschitz manifold, while the locally Lipschitz extension is controlled by an $\varepsilon$-homotopy, hence representing an $\varepsilon$-approximation as well. For the real line, one can simply take a linear homotopy $H: X \times[0,1] \rightarrow \mathbb{R}$ between $f$ and $\varphi$, namely $H(x, t)=(1-t) \cdot f(x)+t \cdot \varphi(x)$. Then $|H(x, t)-\varphi(x)|<\varepsilon(x)$, for every $x \in X$ and $t \in[0,1]$. In fact, Theorem 5.2 is also a natural generalisation of Theorem 4.1 because by Tietze's extension theorem [41], each continuous function $g: A \rightarrow \mathbb{R}$, defined on a closed subset $A$ of a metrizable space $X$, can be extended to a continuous function $\varphi: X \rightarrow \mathbb{R}$.

A word should be also said about the role of the function $\varepsilon: X \rightarrow(0,+\infty)$. For a metric space $(Y, \rho)$, a natural generalisation of the uniform topology on the function space $C(X, Y)$ of all continuous maps from $X$ to $Y$ is the fine topology. It is generated by all sets $\{g \in C(X, Y): \rho(g(x), f(x))<\varepsilon(x), x \in X\}$, where $f \in C(X, Y)$ and $\varepsilon$ runs on $C(X,(0,+\infty))$. This topology was first introduced by Whitney [44], and still sometimes carries his name. In contrast to the uniform topology, the fine topology does not depend on the metric on $Y$ provided $X$ is countably paracompact and normal [13]; for a paracompact $X$, this was previously obtained by Whitehead [42] and Krikorian [27]. Thus, for a metric space $(X, d)$, Theorem 5.2 shows that the locally Lipschitz members of $C(X, \mathbb{R})$ are dense in 
$C(X, \mathbb{R})$ with respect not only to the uniform topology, but also with respect to the fine topology.

The proof of Theorem 5.2 is based on its special case for the uniform topology. The fact that each continuous function on a metric space can be uniformly approximated by locally Lipschitz functions was obtained by several authors, see for instance Miculescu [33, Theorem 2] and Garrido and Jaramillo [20, Corollary 2.8]. Below, we present a simple and self-contained proof of this property.

Proposition 5.3. Whenever $(X, d)$ is a metric space, each continuous function $\varphi: X \rightarrow \mathbb{R}$ is a uniform limit of locally Lipschitz functions.

Proof. If $\varphi: X \rightarrow \mathbb{R}$ is continuous and $\varepsilon>0$, then $\left\{\varphi^{-1}(\mathbf{O}(r, \varepsilon)): r \in \mathbb{Q}\right\}$ is a countable open cover of $X$. Hence, by Proposition 4.2 , it has a locally finite index-subordinated partition of unity $\left\{\xi_{r}: r \in \mathbb{Q}\right\}$ consisting of locally Lipschitz functions. As in Remark 4.7, we may define a locally Lipschitz function $f: X \rightarrow \mathbb{R}$ by $f(x)=\sum_{r \in \mathbb{Q}} \xi_{r}(x) \cdot r, x \in X$. If $x \in X$, then the set $\sigma_{x}=\left\{r \in \mathbb{Q}: \xi_{r}(x) \neq 0\right\}$ is finite. Since $\sum_{r \in \sigma_{x}} \xi_{r}(x)=1$, this implies that

$$
\begin{aligned}
|f(x)-\varphi(x)| & =\left|\sum_{r \in \sigma_{x}} \xi_{r}(x) \cdot r-\sum_{r \in \sigma_{x}} \xi_{r}(x) \cdot \varphi(x)\right| \\
& \leq \sum_{r \in \sigma_{x}} \xi_{r}(x) \cdot|r-\varphi(x)|<\sum_{r \in \sigma_{x}} \xi_{r}(x) \cdot \varepsilon=\varepsilon .
\end{aligned}
$$

The property in Proposition 5.3 can be easily extended from a constant $\varepsilon>0$ to a (lower semi-) continuous function $\varepsilon: X \rightarrow(0,+\infty)$.

Corollary 5.4. Let $(X, d)$ be a metric space and $\varphi: X \rightarrow \mathbb{R}$ be a continuous function. Then for every continuous function $\varepsilon: X \rightarrow(0,+\infty)$, there exists a locally Lipschitz function $f: X \rightarrow \mathbb{R}$ with $|f(x)-\varphi(x)|<\varepsilon(x)$, for every $x \in X$.

Proof. Consider the open cover $O_{n}=\left\{x \in X: \varepsilon(x)>\frac{1}{n}\right\}, n \in \mathbb{N}$, of $X$ and take a locally finite index-subordinated partition of unity $\left\{\xi_{n}: n \in \mathbb{N}\right\}$ consisting of locally Lipschitz functions. Also, by Proposition 5.3, for each $n \in \mathbb{N}$ take a locally Lipschitz function $f_{n}: X \rightarrow \mathbb{R}$ such that $\left|f_{n}(x)-\varphi(x)\right|<\frac{1}{n}, x \in X$. Then the function $f(x)=\sum_{n=1}^{\infty} \xi_{n}(x) \cdot f_{n}(x), x \in X$, is as required because $x \in O_{n}$ implies that $\left|f_{n}(x)-\varphi(x)\right|<\varepsilon(x)$.

Proof of Theorem 5.2. We can use the idea in the proof of Corollary 4.5. Namely, by Theorem $4.1, g$ can be extended to a locally Lipschitz function $\Psi: X \rightarrow \mathbb{R}$. Then $U=\{x \in X:|\Psi(x)-\varphi(x)|<\varepsilon(x)\}$ is an open set containing $A$. Hence, there is a locally Lipschitz function $\eta: X \rightarrow[0,1]$ such that $X \backslash U=\eta^{-1}(0)$ and $A=\eta^{-1}(1)$, see the proof of Corollary 4.5. Moreover, by Corollary 5.4, there is a locally Lipschitz function $\Phi: X \rightarrow \mathbb{R}$ such that $|\Phi(x)-\varphi(x)|<\varepsilon(x)$, for every 
$x \in X$. Finally, define $f: X \rightarrow \mathbb{R}$ by $f(x)=\eta(x) \cdot \Psi(x)+(1-\eta(x)) \cdot \Phi(x), x \in X$. This $f$ is as required.

We conclude this paper with a result about uniform Lipschitz-like approximations of uniformly continuous functions. To this end, let us recall two other classes of "locally" Lipschitz functions. For metric spaces $(X, d)$ and $(Y, \rho)$, a map $f: X \rightarrow Y$ is called uniformly locally Lipschitz if there exists $\delta>0$ such that $f \uparrow \mathbf{O}(x, \delta)$ is Lipschitz, for each $x \in X$. A map $f: X \rightarrow Y$ is called Lipschitz in the small [30] if there exists $\delta>0$ and $K>0$ such that each restriction $f \uparrow \mathbf{O}(x, \delta)$ is $K$-Lipschitz, for every $x \in X$. There are standard examples showing that these Lipschitz-like maps are different. For instance, $\frac{1}{t}:(0,+\infty) \rightarrow \mathbb{R}$ is locally Lipschitz but not uniformly locally Lipschitz, while the function $t^{2}: \mathbb{R} \rightarrow \mathbb{R}$ is uniformly locally Lipschitz but not Lipschitz in the small. Also, one can easily construct Lipschitz in the small functions which are not Lipschitz. For instance, such a function is the one defined in Remark 2.6. This is not surprising because in the realm of bounded maps $f: X \rightarrow Y$, Lipschitz is the same as Lipschitz in the small, see [30, 2.15] and Proposition 3.1.

The Lipschitz in the small maps are naturally related to uniform approximations of uniformly continuous maps.

Proposition 5.5. Let $(X, d)$ and $(Y, \rho)$ be metric spaces and $\varphi: X \rightarrow Y$ be a map which is a uniform limit of Lipschitz in the small maps. Then $\varphi$ is uniformly continuous.

Proof. Let $\varepsilon>0$ and $f: X \rightarrow Y$ be a map which is Lipschitz in the small such that $\rho(f(x), \varphi(x))<\frac{\varepsilon}{3}$ for every $x \in X$. By definition, there are $\delta>0$ and $K>0$ such that $f \uparrow \mathbf{O}(x, \delta)$ is $K$-Lipschitz, for every $x \in X$. Then taking $\delta_{0}=\min \left\{\frac{\varepsilon}{3 K}, \delta\right\}$, it follows that $\rho(\varphi(x), \varphi(z))<\varepsilon$ for every $x, z \in X$ with $d(x, z)<\delta_{0}$.

In case $Y=\mathbb{R}$ is the real line, the converse is also true. The fact that each uniformly continuous function $\varphi: X \rightarrow \mathbb{R}$ is a uniform limit of Lipschitz in the small functions was observed by several authors. For a compact metric space $X$, the result was obtained by Georganopoulos [22]. For a bounded uniformly continuous function $\varphi: X \rightarrow \mathbb{R}$, the result can be found in [25, Theorem 6.8], see also [34, Theorem 1]. In fact, the proof in [25] is very simple and consists of showing that the sequence of Lipschitz functions defined as in (2.1) for $\kappa=n \in \mathbb{N}$ is uniformly convergent to $\varphi$. For an arbitrary uniformly continuous function, the result was obtained by Garrido and Jaramillo [21, Theorem 1]. Subsequently, a simple proof of this theorem was given by Beer and Garrido in [9, Theorem 6.1], which was a refined version of the argument in [25]. Below we simplify further this proof illustrating its natural relationship with Theorem 2.1.

Theorem 5.6. If $(X, d)$ is a metric space, then each uniformly continuous function $\varphi: X \rightarrow \mathbb{R}$ is a uniform limit of functions which are Lipschitz in the small. 
Proof. Suppose that $\varphi: X \rightarrow \mathbb{R}$ is uniformly continuous and $\varepsilon>0$. Also, let $\delta>0$ be such that $|\varphi(x)-\varphi(y)|<\varepsilon$, for every $x, y \in X$ with $d(x, y)<2 \delta$. Next, following [9], take $k \in \mathbb{N}$ such that $k \delta>\varepsilon$, and define $f: X \rightarrow \mathbb{R}$ by

$$
f(x)=\inf _{y \in \mathbf{O}(x, 2 \delta)}[\varphi(y)+k d(y, x)], \quad x \in X .
$$

Then $\varphi(x) \geq f(x) \geq \varphi(x)-\varepsilon$ because $\varphi(y)>\varphi(x)-\varepsilon$ for every $y \in \mathbf{O}(x, 2 \delta)$, so $|f(x)-\varphi(x)|<\varepsilon$. Whenever $y \in \mathbf{O}(x, 2 \delta)$ with $d(y, x) \geq \delta$, since $k \delta>\varepsilon$, it follows that $f(x) \leq \varphi(x)<\varphi(y)+\varepsilon<\varphi(y)+k \delta \leq \varphi(y)+k d(y, x)$. Therefore, we actually have that

$$
f(x)=\inf _{y \in \mathbf{O}(x, \delta)}[\varphi(y)+k d(y, x)], \quad x \in X .
$$

This implies that $f$ is $k$-Lipschitz on each open $\frac{\delta}{2}$-ball. Indeed, take $p, q \in X$ with $d(p, q)<\delta$. Then $\mathbf{O}(p, \delta) \cup \mathbf{O}(q, \delta) \subset \mathbf{O}(p, 2 \delta) \cap \mathbf{O}(q, 2 \delta)=X_{p q}$. Since $k d(x, p) \leq k d(x, q)+k d(q, p), x \in X_{p q}$, as in the proof of Theorem 2.1 but now using (5.2) and (5.3), we get that $f(p) \leq f(q)+k d(p, q)$. Evidently, this is equivalent to $|f(p)-f(q)| \leq k d(p, q)$.

\section{References}

[1] R. Baire, Sur les fonctions de variables réelles., Annali di Mat. (3) 3 (1899), 1-123 (French).

[2] _ Nouvelle démonstration d'un théorème sur les fonctions discontinues, Bull. Soc. Math. France 28 (1900), 173-179.

[3] _ Sur les séries à termes continus et tous de même signe, Bull. Soc. Math. France 32 (1904), 125-128.

[4] _ Leçons sur les fonctions discontinues, Les Grands Classiques Gauthier-Villars. [Gauthier-Villars Great Classics], Éditions Jacques Gabay, Sceaux, 1995, Reprint of the 1905 original.

[5] S. Banach, Sur les fonctionelles linéaires. I., Stud. Math. 1 (1929), 211-216 (French).

[6] _ Sur les fonctionelles linéaires. II., Stud. Math. 1 (1929), 223-239 (French).

[7] _ Théorie des opérations linéaires, Monografie Matematyczne, Warszawa, 1932 (French).

[8] _ Theory of linear operations, North-Holland Mathematical Library, vol. 38, NorthHolland Publishing Co., Amsterdam, 1987, Translated from the French by F. Jellett, With comments by A. Pełczyński and Cz. Bessaga.

[9] G. Beer and M. I. Garrido, Locally Lipschitz functions, cofinal completeness, and UC spaces, J. Math. Anal. Appl. 428 (2015), no. 2, 804-816.

[10] Ştefan Cobzaş, R. Miculescu, and A. Nicolae, Lipschitz functions, Lect. Notes Math., vol. 2241, Cham: Springer, 2019 (English).

[11] J. Czipszer and L. Gehér, Extension of functions satisfying a Lipschitz condition, Acta Math. Acad. Sci. Hungar. 6 (1955), 213-220.

[12] G. De Marco and R. G. Wilson, Realcompactness and partitions of unity, Proc. Amer. Math. Soc. 30 (1971), 189-194.

[13] G. Di Maio, L. Holá, D. Holý, and R. A. McCoy, Topologies on the space of continuous functions, Topology Appl. 86 (1998), 105-122.

[14] C. H. Dowker, On countably paracompact spaces, Canad. J. Math. 3 (1951), 291-224. 
[15] J. Dugundji, Topology, Boston, 1966.

[16] E. Durand-Cartagena and J. A. Jaramillo, Pointwise Lipschitz functions on metric spaces, J. Math. Anal. Appl. 363 (2010), no. 2, 525-548.

[17] R. Engelking, General topology, revised and completed edition, Heldermann Verlag, Berlin, 1989.

[18] A. Fathi, Partitions of unity for countable covers, Amer. Math. Monthly 104 (1997), no. 8, $720-723$.

[19] Z. Frolík, Existence of $l_{\infty}$-partitions of unity, Rend. Sem. Mat. Univ. Politec. Torino 42 (1984), no. 1, 9-14 (1985).

[20] M. I. Garrido and J. A. Jaramillo, Homomorphisms on function lattices, Monatsh. Math. 141 (2004), no. 2, 127-146.

[21] _ Lipschitz-type functions on metric spaces, J. Math. Anal. Appl. 340 (2008), no. 1, $282-290$.

[22] G. Georganopoulos, Sur l'approximation des fonctions continues par des fonctions lipschitziennes, C. R. Acad. Sci. Paris Sér. A-B 264 (1967), A319-A321.

[23] H. Hahn, Über lineare Gleichungssysteme in linearen Räumen., J. Reine Angew. Math. 157 (1927), 214-229 (German).

[24] F. Hausdorff, Über halbstetige Funktionen und deren Verallgemeinerung, Math. Zeitschr. 5 (1919), 292-309.

[25] J. Heinonen, Lectures on analysis on metric spaces, Universitext, Springer-Verlag, New York, 2001.

[26] E. Helly, Über lineare Funktionaloperationen., Wien. Ber. 121 (1912), 265-297 (German).

[27] N. Krikorian, A note concerning the fine topology on function spaces, Compos. Math. 21 (1969), 343-348.

[28] S. Lefschetz, Algebraic Topology, American Mathematical Society Colloquium Publications, v. 27, American Mathematical Society, New York, 1942.

[29] J. Luukkainen and J. Väisälä, Elements of Lipschitz topology, Ann. Acad. Sci. Fenn. Ser. A I Math. 3 (1977), no. 1, 85-122.

[30] J. Luukkainen, Rings of functions in Lipschitz topology, Ann. Acad. Sci. Fenn. Ser. A I Math. 4 (1979), no. 1, 119-135.

[31] J. Malý, A simple proof of the Stepanov theorem on differentiability almost everywhere, Exposition. Math. 17 (1999), no. 1, 59-61.

[32] E. J. McShane, Extension of range of functions, Bull. Amer. Math. Soc. 40 (1934), no. 12, 837-842.

[33] R. Miculescu, Approximation of continuous functions by Lipschitz functions, Real Anal. Exchange 26 (2000/01), no. 1, 449-452.

[34] — Approximations by Lipschitz functions generated by extensions, Real Anal. Exchange 28 (2002/03), no. 1, 33-40.

[35] H. Rademacher, Über partielle und totale differenzierbarkeit von Funktionen mehrerer Variabeln und über die Transformation der Doppelintegrale, Math. Ann. 79 (1919), no. 4, 340359.

[36] M. E. Rudin, Dowker spaces, Handbook of set-theoretic topology, North-Holland, Amsterdam, 1984, pp. $761-780$.

[37] C. H. Scanlon, Rings of functions with certain Lipschitz properties, Pacific J. Math. 32 (1970), 197-201.

[38] W. Stepanoff, Über totale Differenzierbarkeit, Math. Ann. 90 (1923), no. 3-4, 318-320.

[39] V. V. Stepanov, Sur les conditions de l'existence de la différentielle totale., Rec. Math. Moscou 32 (1925), 511-527 (French). 
[40] A. H. Stone, Paracompactness and product spaces, Bull. Amer. Math. Soc. 54 (1948), 977982.

[41] H. Tietze, Über Funktionen, die auf einer abgeschlossenen Menge stetig sind, J. für die reine und angew. Math. 145 (1915), 9-14.

[42] J. H. C. Whitehead, Manifolds with transverse fields in euclidean space, Ann. of Math. (2) 73 (1961), 154-212.

[43] H. Whitney, Analytic extensions of differentiable functions defined in closed sets, Trans. Amer. Math. Soc. 36 (1934), no. 1, 63-89.

[44] _ Differentiable manifolds, Ann. of Math. (2) 37 (1936), 645-680.

Department of Mathematics, Faculty of Science, University of Malta, Msida MSD 2080, MALTA

E-mail address: valentin.gutev@um.edu.mt 